\title{
Kratos, les mythes, le monde. Jeux vidéo et représentations sociales contemporaines de la mythologie
}

Kratos, between world and mythology. Video games and contemporary social representations of mythology

\section{Adrien Coignoux et Yohann Guffroy}

\section{(2) OpenEdition Journals}

Édition électronique

URL : https://journals.openedition.org/sdj/3150

DOI : $10.4000 /$ sdj.3150

ISSN : 2269-2657

\section{Éditeur}

Laboratoire EXPERICE - Centre de Recherche Interuniversitaire Expérience Ressources Culturelles Education

\section{Référence électronique}

Adrien Coignoux et Yohann Guffroy, « Kratos, les mythes, le monde. Jeux vidéo et représentations sociales contemporaines de la mythologie », Sciences du jeu [En ligne], 15 | 2021, mis en ligne le 20 juin 2021, consulté le 26 novembre 2021. URL : http://journals.openedition.org/sdj/3150 ; DOI : https:// doi.org/10.4000/sdj.3150

Ce document a été généré automatiquement le 26 novembre 2021.

\section{(c) (1) ()}

La revue Sciences du jeu est mise à disposition selon les termes de la Licence Creative Commons Attribution - Pas d'Utilisation Commerciale - Pas de Modification 4.0 International. 


\section{Kratos, les mythes, le monde. Jeux vidéo et représentations sociales contemporaines de la mythologie}

Kratos, between world and mythology. Video games and contemporary social representations of mythology

Adrien Coignoux et Yohann Guffroy

1 Développée par David Jeffet et Cory Barlog au sein du SCE Santa Monica Studio, God of War comprend aujourd'hui huit jeux sur différentes plateformes, dont une première trilogie sur Playstation 2 et $3(2005,2007,2010)$ et un dernier sans numéro ni sous-titre sur Playstation 4 et sorti en mai 2018. ${ }^{1} \mathrm{Si}$ tous les jeux ont été accueillis avec enthousiasme par les joueurs, le premier s'étant vendu à près de 4,6 millions d'exemplaires l'année de sa sortie, c'est le dernier qui obtient le plus grand succès. L'opus s'est en effet vendu à 3 millions d'exemplaires en seulement trois jours pour atteindre un total de 10 millions au cours de sa première année. ${ }^{2}$

Outre son succès commercial, ce dernier volet se distingue également par un changement de "paysage mythologique». Par cette expression, nous entendons l'emprunt d'éléments appartenant à une mythologie basée sur un système polythéiste associé à une aire culturelle précise, à des fins narratives et esthétiques, sans en respecter tous les enjeux mais se référant suffisamment à ses logiques de base pour que ce système soit plus qu'un simple "arrière-plan ». Dans le cas de God of War, alors que dans les sept premiers opus le personnage principal, Kratos, évolue au sein de l'univers mythologique grec antique, les développeurs ont pris le parti pour le dernier jeu de changer complètement d'environnement en optant pour les pays nordiques et leur mythologie. Il s'agit là d'un exemple de ce que Luca Arruns Panaro qualifie de «tournant nordique » initié il y a une quinzaine d'années dans la culture de masse. En se référant à ce même épisode de God of War, il note «qu'un changement si radical [de paysage mythologique] basé sur une mode montre le pouvoir d'attraction qu'exerce actuellement le "Nord" dans la culture et la société » (Panaro, 2019, p. 51). Toutefois, cette rupture spatiale ne s'accompagne pas d'une rupture de la trame narrative: 
comme dans les trois premiers opus, les aventures de Kratos l'amènent à entrer en conflit avec les principales divinités de l'aire culturelle dans laquelle il évolue, se concluant le plus souvent par leur mort. Après avoir tué Arès dont il prend la place en tant que dieu de la guerre et de nombreuses divinités grecques, jusqu'à Zeus lui-même, dans la première trilogie, c'est au tour des divinités nordiques, à commencer par Baldr, d'être l'objet de son courroux. Seul diffère le moteur de la narration: alors que les premières aventures de Kratos reposent sur sa quête de rédemption - il a causé la mort de sa femme et sa fille - les dernières sont centrées sur l'éducation de son fils, Atreus.

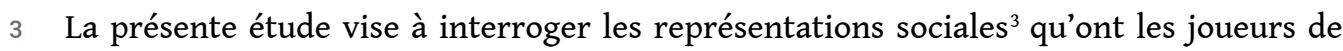
God of War de ce qu'est une mythologie à partir de leurs réactions à ce changement de paysage. Pour ce faire, nous avons choisi parmi les multiples approches possibles (Wagner, 2012 ; Bainbridge, 2013 ; Campbell, Price Grieve, 2014 ; Di Filippo, 2016a) de croiser les analyses de seize fils de discussion répartis entre 2018 et 2020 sur deux forums distincts, Reddit (anglophone) et Jeuxvideo.com (francophone) et d'une partie du contenu du jeu. Ces fils totalisent plus de six cent cinquante messages plus ou moins courts. Ces réactions sont interprétées à la lumière des travaux de spécialistes du jeu vidéo, d'historiens des religions ou encore d'anthropologues, afin de faire apparaître toute la spécificité de l'idée de mythologie transparaissant dans les diverses réactions. Notre analyse s'inscrit donc à la fois dans l'étude des représentations sociales telle que Serge Moscovici (1961) l'a théorisée, mais aussi de l'esthétique de la réception (Iser, 1985 [1976]; Jauss, 2019 [1978]) en ce qu'elle s'intéresse aux «conditions sociales d'émergence et de constitution de l'interprétation » (Saemmer, 2016).

4 Notre analyse s'organise en quatre temps. Il s'agira d'abord d'étudier le positionnement argumentatif des joueurs sur les forums de discussion afin de comprendre la manière dont ils prennent part au débat. Nous proposerons ensuite une rapide présentation de l'état actuel de la définition académique des termes «mythe » et « mythologie » afin de les confronter aux représentations des joueurs. Celles-ci nous permettront de développer les deux dernières étapes de notre réflexion centrée sur l'interprétation des deux principaux pôles de réaction des joueurs. Le premier concerne l'impossibilité pour certains joueurs de voir coexister dans le même espace deux systèmes polythéistes et posent alors d'un côté la question de la cohérence scénaristique de l'autre, plus profondément, celle d'une représentation particulière du monde. Le second porte, lui, sur l'introduction de Kratos au sein du panthéon nordique. Dans la mesure où les développeurs ont souhaité qu'il ait un impact sur son environnement d'arrivée, ces derniers ont dû adapter la tradition médiévale scandinave à leur récit, ce que n'ont pas manqué de remarquer et commenter les joueurs.

\section{Le forum comme lieu d'expression du métajeu}

Le forum est le plus souvent questionné pour sa forme a priori d'espace public au sein duquel tout un chacun peut s'exprimer (Flichy, 2008; Chaker, 2017). Moyennant l'acceptation des règles de participation, chaque utilisateur échange sur un pied d'égalité avec les autres participants. La conversation et la confrontation d'idées qui s'établissent peuvent alors être perçues en tant que forme d'apprentissage que Grégory Bateson définit comme " un changement dans la spécificité de la réponse ou dans le processus même de l'apprentissage, à travers une correction des erreurs de choix à l'intérieur d'un ensemble de possibilités » (Bateson, 1977 dans Pledel, 2007, p. 277). Si, 
de manière générale, le forum a une fonction de support, de balise (Peeters, Charlier, 1999), c'est encore plus vrai dans le domaine du jeu vidéo. Il devient pour le joueur un élément précieux parmi la boîte à outils du « hors jeu », défini comme « l'ensemble des espaces d'information et de communication dédiés aux joueurs, focalisés sur l'activité de jeu, hors de l'espace logiciel» (Zabban, 2009, p. 82). Dans le cas qui nous occupe ici, le forum n'est pas étudié à travers le prisme de l'assistance ou de la collaboration entre joueurs dans le but de réaliser une tâche mais à travers celui de l'échange et de la réflexion. Il se voit alors doté d'une seconde fonction, celle de support de développement $\mathrm{du}$ "métajeu» défini par Richard Garfield comme «ce qui fait l'interface entre un jeu et la vie » (Garfield, 2000 dans Zabban, 2014, p. 13). En effet, tous les sujets de discussion sur les forums ne sont pas des appels à l'aide mais sont parfois des interrogations plus réflexives sur le contenu même du jeu, sur sa pertinence ou encore sa cohérence.

6 La mythologie constitue le paysage de fond de la licence God of War et est l'objet de débats, bien qu'elle n'influe pas sur la manière de jouer du joueur. De ce point de vue, cette thématique entre pleinement dans la définition du métajeu puisque, par l'intermédiaire des échanges entre joueurs, elle est extraite du jeu et amenée au plus près de l'expérience quotidienne pour être disséquée, voire objectivée. Ce n'est pas la manière de jouer dont il est question mais ce qui est raconté. En cela, les utilisateurs sont dans un processus de «forage » du jeu (Jenkins, 2009), cherchant à dénouer tous les fils de la trame narrative pour en toucher l'essence.

7 L'utilisation des forums Reddit et Jeuxvideo.com ne permet malheureusement pas d'établir les profils sociologiques des joueurs. Le forum anglophone ne donne effectivement aucune visibilité aux caractéristiques telles que l'âge, la provenance géographique, le genre, ou encore une description de soi et ces champs sont trop peu renseignés par les internautes sur Jeuxvideo.com pour être exploitables. Nous n'avons par ailleurs pas pu lancer d'enquête auprès de chaque participant. Il est en revanche possible, à partir des messages des utilisateurs, de mettre en lumière les diverses postures adoptées lorsqu'il s'agit de discuter de mythologie et qui peuvent être regroupées en trois catégories : (1) la critique par l'avis personnel ; (2) l'appui sur des jeux externes; (3) l'utilisation de références littéraires, académique ou non. On fera tout de suite remarquer que ces stratégies argumentatives ne sont pas explicitement hiérarchisées par les joueurs, laissant plutôt penser que les discussions s'ancrent dans l'un des pôles argumentatifs relevés par Ruth Amossy $(2012,818)$ au sein duquel certains discours "infléchissent des façons de penser, de voir et de sentir sans se donner explicitement comme une entreprise de persuasion ».

8 La grande majorité des participants s'expriment à travers diverses formulations du type "je pense que", "je souscris à», "c'est pourquoi je me dis que », etc. Ils ne s'appuient explicitement sur aucune source mais ils évoquent plutôt une réflexion personnelle, une expérience du jeu ou bien des connaissances non situées qui ne permettent pas de déterminer où et quand elles ont été acquises. Nous rapportons ici deux brefs exemples : «Il me semble que la mythologie grecque (et ce même dans [1]e jeu) englobait pas juste une région mais le monde tout entier [...] $»^{4}$ ou encore celui-ci, "Désolé si j'offense les sensibilités de quelqu'un mais plus je réfléchis, plus je me dis que les religions païennes sont plus logiques que le monothéisme. [...] $» .^{5}$ Dans ces deux cas, les auteurs n'utilisent aucun support argumentatif, entendu comme une référence qui servirait de soutien au discours et qu'il faudrait s'employer à réfuter en plus de 
l'avis du participant. Ici, ils reconnaissent implicitement que ce qu'ils énoncent «n'est pas une évidence "inconditionnellement et absolument valable", mais une prise de position discutable, raisonnée et subjective » (Koren, 2008, \$17).

9 Si l'avis personnel prédomine, certains cherchent à appuyer leur réflexion par l'introduction de références extérieures au jeu. Des joueurs font notamment part de leur gêne à l'idée que puissent coexister dans un même espace deux genèses et deux fins du monde :» Le problème de la coexistence des mythologies concerne uniquement la genèse. Le monde a-t-il été créer ${ }^{6}$ par le cadavre d'Ymir (nordique) ou par Gaïa (grecque) ?».7 En réponse, un internaute rappelle que le jeu Age of Mythology (Microsoft), sorti en 2002 et basé sur les principes de la licence Age of Empire, reposait sur l'affrontement de civilisations, chacune soutenue par un panthéon particulier :

Ou peut-être que le monde selon chaque mythologie se limite au frontière du pays, il n'y a rien d'improbable là-dedans. [...] Le jeu Age of Mythology a développé la même idée il y a 15 ans, il n'y a pas eu de débat sur ça. Il est même devenu culte grâce à cette idée. ${ }^{8}$

Dans la même veine, certains recommandent d'autres jeux afin d'éviter d'avoir à recourir à des sources d'époque ou de la littérature académique. Par exemple, le jeu Hellblade: Senua's Sacrifice (Ninja Theory, 2017) est pour les joueurs une référence vers laquelle se tourner si l'on souhaite poursuivre la découverte de la mythologie nordique. Ce dernier retrace l'aventure d'une guerrière picte à la recherche de l'âme de son amant retenue à Helheim, le monde des morts, à la manière du mythe d'Orphée. Accompagnée de l'esprit d'un esclave nordique, son voyage lui fait combattre diverses entités mythologiques telles que le corbeau Valravn ou encore le géant Surtr. Ce jeu donne notamment la possibilité pour les joueurs de comparer la mise en représentation de divers éléments de la mythologie nordique avec celle de God of War. À titre d'exemple, l'une des mystérieuses figures que croise Kratos à Helheim est un gigantesque oiseau non identifié et qui interroge beaucoup les joueurs qui se divisent en deux groupes : d'un côté ceux qui considèrent qu'il s'agit du géant Hræsvelg, auteur de tous les vents', et de l'autre ceux qui le prennent pour l'un des trois oiseaux annonciateurs du Ragnarök ${ }^{10}$. De même, dans Hellblade, on peut apercevoir dans le décor un gigantesque oiseau de bois, ce qui permet la transposition des questionnements énoncés dans God of War. Les joueurs s'interrogent et échafaudent les mêmes théories, mais mettent surtout en parallèle les jeux pour tenter de comprendre la référence qui se cache derrière la présence des deux entités. Pour l'un des internautes, ces deux éléments représentent la même entité, ajoutant que « Hellblade est symbolique alors que God of War est littéral [dans leur mise en scène des références] ». ${ }^{11}$

11 Enfin, la dernière stratégie identifiée est de faire appel à des références littéraires. Dans l'un des fils francophones, un internaute se réfère à une interview retranscrite des développeurs du studio pour appuyer son propos ${ }^{12}$. Sur Reddit, alors qu'il est question, dans un fil de la possibilité d'introduire de nouvelles mythologies dans le jeu, des références inattendues apparaissent dans les sous-conversations. Les joueurs se réfèrent par exemple à la Bhagavad-Gita ${ }^{13}$, texte qui prend de l'importance dans la contre-culture $\mathrm{du} \mathrm{xx}^{\mathrm{e}}$ siècle, pour justifier la plausibilité d'une rencontre entre Kratos et le dieu Krishna :

Je pense que Kratos rencontrant Krishna pourrait faire bien plus sens [que rencontrer Jésus] dans la mesure où la Baghavad-Gita tourne autour de l'enseignement de Krishna au prince Arjuna sur la manière essentielle de «gagner la guerre » qui est la toile de fond de l'histoire [du jeu] [;] mais c'est surtout 
symbolique pour une machine à tuer [Human Killing Machine] qui lutterait contre elle-même pour atteindre l'illumination et la transcendance de la mort afin de trouver Dieu. ${ }^{14}$ œuvres. En effet, dans la première trilogie, Kratos est un anti-héros tueur de dieux qui est en quête de rédemption pour avoir assassiné sa propre famille. La notion de transcendance de la mort pour trouver la paix, et non «Dieu ", fait l'objet du nouvel opus. À la suite du message cité précédemment, lui-même sous-réponse d'une discussion plus générale, s'engage un long échange sans issue sur la prédominance et permanence de Jésus par rapport aux religions polythéistes. Les utilisateurs citent pêlemêle les Évangiles, mais aussi Platon qui est mentionné pour ses réflexions sur le "péché » (Bianchi, 1966) sans qu'aucune œuvre ne soit cependant spécifiquement évoquée. On remarque donc que la discussion générale qui partait d'une question globale sur une possibilité narrative du jeu tourne à une discussion théologique sur la prédominance d'une religion sur une autre. Ces échanges se retrouvent notamment dans d'autres fils où les internautes discutent de la pertinence d'orienter le jeu vers les récits présents dans l'Ancien et le Nouveau Testament et ses possibilités de mise en scène $e^{15}$. Cela rejoint parfaitement la définition donnée plus haut du métajeu par Garfield, « ce qui fait l'interface entre le jeu et la vie ». Le forum est bien ici un lieu où se négocie une définition partagée du monde (Zabban, 2014).

D'autres, plutôt en quête d'une littérature "simple d'accès ", s'engagent alors dans un apprentissage tangentiel dans le but de se renseigner davantage sur la mythologie nordique pendant ou après la pratique du jeu (Mozelius, Fagerström, Söderquist, 2017 ; Dussarps, 2020). ${ }^{16}$ On retrouve alors de nombreux commentaires, anglais comme français, recommandant la lecture du livre La Mythologie viking de Neil Gaiman (2017), un ouvrage qui offre une version romancée des histoires des dieux nordiques. ${ }^{17}$ D'autres, plutôt anglais cette fois-ci, proposent The Gospel of Loki de Joanne Harris (2014) qui donne, ici aussi de manière romancée, l'histoire des dieux nordiques du point de vue de Loki, depuis sa naissance jusqu'à sa mort. ${ }^{18}$ Moins nombreux sont ceux qui proposent une lecture des textes canoniques des $\mathrm{X}^{\mathrm{e}}$-XIII ${ }^{\mathrm{e}}$ siècles tels que l'Edda en prose de Snorri Sturluson (1991) ou l'Edda poétique (Boyer, 1992) même si certains souhaitent en prendre connaissance afin d'être plus à même de critiquer le jeu : «Je suis en train de le lire [l'Edda précis n'est pas indiqué] pour mieux critiquer après la transposition mythologique scandinave faite a la truelle sur ce jeu [...]. $»^{19}$ On notera que la Völuspa ${ }^{20}$ n'est citée qu'une fois parmi les différents fils et que la littérature académique est absente. Ces remarques ne valent toutefois que pour les communautés God of War de Reddit et Jeuxvideo.com dans la mesure où il en existe une autre constituée autour d'une section différente du forum anglophone intitulée « Norse » qui propose pour sa part des ouvrages scientifiques sur la question mythologique. De rares internautes renvoient aux discussions qui s'y développent.

La mise en lumière de ces différentes stratégies montre que peu de joueurs font explicitement appel aux textes médiévaux scandinaves pour défendre leur point de vue, voire même discuter celui des autres intervenants, préférant le plus souvent des écrits intermédiaires plus contemporains et romancés. Il semble qu'il y ait une base de connaissances tacites commune aux participants. Dans tous les échanges que nous avons étudiés, les joueurs se situent dans un monde " profane » (Becker, 2016, p. 74) où personne n'adopte une posture plus docte que les autres. Ils évoluent tous au sein de la même sphère non académique, certes à des degrés de connaissances différents, mais 
aucun de plus "savant» n'essaye de guider les autres vers une réponse, une interprétation qui serait jugée "vraie », à la différence de la figure du gourou chez Howard Becker (2016, p. 75-79). La faible proportion des joueurs faisant appel aux textes médiévaux scandinaves montre que la connaissance des sources historiques est loin d'être un prérequis pour échanger sur la mythologie nordique, rejoignant notamment ce que soutient Laurent Di Filippo (2016a, p. 450) dans son étude sur le jeu Age of Conan. Cette non mobilisation des sources n'empêche cependant pas les joueurs de discuter et remettre en question les choix des développeurs quant à l'orientation prise en ce qui concerne la mise en scène de cette mythologie et les implications qui en découlent.

\section{« Mythologie »: Un point d'ancrage académique}

15 Il est ainsi tout à fait intéressant de constater le décalage entre les problématiques mises en exergue par la trame narrative de la licence à travers le changement de paysage mythologique et les principaux sujets de réaction des joueurs sur les fils de discussion étudiés. Là où les scénaristes s'emparent du thème des écarts entre statuts humain et divin ainsi que les comportements qui leurs sont associés (à travers le récit des aventures d'un «tueur de dieux »), les joueurs se saisissent pour leur part, sur dix des seize fils, de ce qu'ils pensent être des incohérences induites par le changement de support mythologique. L'usage de la mythologie, et plus largement de la religion, a beau être dans le jeu à visée "référentielle » (Bosman, 2016, p. 35), il n'en attire pas moins l'attention des joueurs. Mais tout bien considéré, ce décalage n'apparaît pas si illogique puisque ce transfert représente la principale rupture entre l'épisode de 2018 et les précédents. Les joueurs étant habitués à une trame narrative traitant les relations entre les humains et les dieux dans une perspective conflictuelle, il n'est pas étonnant de ne la voir prendre qu'une faible place parmi les réactions des internautes. Apparaît alors clairement le rôle du forum en tant que métajeu où se rencontrent le réel et le virtuel autour de questions de représentation de la mythologie.

Les joueurs ayant ressenti le besoin d'exprimer ce trouble se divisent principalement en deux groupes : ceux qui acceptent et cherchent à justifier la plausibilité du transfert et ceux qui relèvent des incohérences rédhibitoires. Une fracture qui selon nous est à relier à des différences de représentation dans la définition d'une "mythologie ", qui, une fois examinées au prisme des connaissances historiques actuelles, nous apparaissent riches d'enseignements. La question des incohérences qu'implique le transfert de paysage mythologique s'articule, dans les fils de discussion étudiés, autour de deux sujets principaux : la question de l'existence de plusieurs systèmes polythéistes dans une même structure narrative et celle de l'identité de Kratos une fois inséré dans le panthéon nordique.

17 Avant toute tentative d'analyse, il nous faut rapidement aborder la question de la définition de la mythologie et ses caractéristiques telles que la proposent les chercheurs en sciences humaines. Elle nous servira de point d'ancrage nous permettant de mettre en perspective les propos des joueurs et donc de faire apparaître la spécificité de leurs représentations à partir de l'écart qui réside entre les caractéristiques qu'ils attribuent aux mythologies et celles qu'y attribuent les chercheurs de ces cinquante dernières années. 

controversées. Utilisées depuis le début du XIXe siècle par les linguistes, anthropologues et philosophes, elles sont devenues des totalités rassemblant un grand nombre de types de récits différents issus de cultures non-modernes comme non-occidentales sur la base de leurs caractéristiques internes, au premier chef la présence d'éléments liés au merveilleux. Elles servirent ainsi longtemps de "figures d'altérité » (Détienne, 1981, p. 94 et 104 ; Borgeaud, 2010, p. 856 ; Calame, 2015, p. 17) afin de désigner, dans une perspective évolutionniste, des modes de compréhension et de représentation du monde opposés à ceux prônés par les Lumières et l'Occident moderne à partir de l'opposition lógos / mûthos et de la conviction en l'existence du «miracle grec» qui permit l'avènement de la Raison comme principal prisme d'explication du monde (Détienne, 1981, p. 27-28; Calame, 2015, p. 26, 32). Mais depuis le début du xxe siècle, plusieurs anthropologues, à commencer par Franz Boas (1914), ont remis en cause ces catégories, faisant apparaître leur dimension artificielle et ethnocentrée (Détienne, 1981, p. 238) ou étique une fois devenues des universaux et ont prôné un retour à une perspective émique (Sardan, 1998) inscrite dans le particulier (Di Filippo, 2020). Ce mouvement critique s'intensifie lorsque les historiens de la Grèce antique, Jean-Pierre Vernant $(1962$; 1974) puis Marcel Détienne (1981), entreprennent de faire l'histoire de ces catégories. Ils ont alors montré qu'à travers les catégories de "mythe » et de «mythologie», les chercheurs occidentaux modernes avaient essentialisé (Calame, 2015, p. 30-31) des pratiques de mise en récits distinctes (issues de contextes culturels radicalement différents) en les réduisant à leurs seules trames narratives dans l'optique d'analyses structuralistes, afin de les comparer.

C'est en vertu de cette critique que Claude Calame (2015) et Sarah Iles Johnston (2018) ont récemment proposé de nouvelles approches des récits que désignent traditionnellement ces catégories. Opposant à la perspective structuraliste et globalisante traditionnelle une approche centrée sur le particulier qu'il nomme "ethnopoétique ", Calame (2015, p. 34) propose d'analyser ces récits à partir de la confrontation de leur contexte original, au sens du contexte d'énonciation extradiscursif qui leur est propre, avec le contexte intra-discursif, celui des récit(s) qu'ils présentent (p.76-78); chaque version d'un même récit est ainsi rapportée à son contexte d'énonciation particulier, la performance poétique (p. 42-43). Cette approche permet de dégager sa «pragmatique » (p. 27), c'est-à-dire son effet sur la réalité où il est exprimé. Si Johnston (2018, p. 12 et 92 ) choisit, elle aussi, d'aborder les récits " mythiques » à partir de leurs conditions d'énonciation originales et de leurs effets sur le réel, la « pragmatique » du récit qu'elle cherche à mettre en évidence est de nature plus générale. Elle n'est plus fondée sur l'effet que chaque récit, dans chacune de ses variantes, induit sur des publics précis mais sur le rôle qu'ils jouent dans leur ensemble sur la conviction qu'ont les différents publics de l'existence des puissances divines (p. 21, 78 et 119).

Toutefois, si certains des outils développés par ces deux chercheurs nous seront utiles dans notre analyse, il existe un écart irréductible entre leur perspective de recherche et la nôtre, celui-là même qui nous pousse à utiliser l'expression de «paysage mythologique ». Car les différents «mythes» et "mythologies» que nous allons aborder apparaissent dans un cadre d'expérience, le jeu vidéo, bien différent de l'activité sociale où ils prennent place originellement, les poésies grecque et nordique. Mais, loin de toute préoccupation sur la question des origines, il s'agit avant tout 
d'étudier une actualisation des différentes matières mythologiques convoquées, une étape du processus que Hans Blumenberg a appelé «le travail sur le mythe » entendu comme les mécanismes de transmission et réception d'une matière en constante évolution (Blumenberg, 2001 [1979]). Dans ce contexte vidéoludique, davantage que des récits avec un véritable effet sur le réel - une "pragmatique » - les mythes que nous étudions constituent alors une "illusion esthétique " ${ }^{21}$ (Wolf, 2013; Panaro, 2019, p. 20-23) au sens où le public de ces récits, les joueurs dans notre cas, a tout à fait conscience que le récit qui lui est présenté est une fiction, une création artificielle qui ne fait qu'imiter le réel sans prétention à la vérité. Cela contrairement au public des performances poétiques antiques dans le cas grec et médiévales dans le cas nordique pour qui ces récits, bien qu'artificiels, renvoyaient néanmoins à des événements prétendus réels (Calame, 2015, p. 92), même si les travaux de Paul Veyne nous ont appris à ne pas appréhender tout phénomène de croyance à l'aune de nos propres conceptions (Veyne, 1983). Privées de leur effet sur le réel, les mythologies convoquées par la série God of War ne sont plus que des «figures d'altérité » dont le principal effet sur le public est d'ordre esthétique et ludique. Le jeu vidéo est donc une illusion esthétique d'un genre un peu particulier car, au-delà de la dimension ludique, réside également une dimension mercantile qui influe sur la forme prise par ce type de création. En effet, comme toute industrie, le jeu vidéo est soumis à des objectifs de rentabilité qui influent sur l'esthétique choisie par les concepteurs. Si les développeurs empruntent ces mythologies, c'est d'une part pour leur exotisme formel qui plonge le joueur dans un monde rempli de dieux et d'autre part, pour leur capacité à rendre le jeu plus immersif et réaliste en usant de mythologies traditionnelles (Cooper, 2016 p. 51). Et ceci avec suffisamment de fidélité pour que cet emprunt constitue, audelà d'un simple arrière-plan, un véritable paysage dont les reliefs (les caractéristiques spécifiques) influent, comme nous le verrons, sur le récit présenté.

21 C'est ainsi au prisme de cette définition "particulariste » (Di Filippo, 2016b, p. 115) de la mythologie et plus largement de l'histoire de l'utilisation des catégories de "mythe " et «mythologie », dont elle est l'une des plus récentes étapes, que nous analyserons les deux principaux pôles de réactions des joueurs. La question de la possible cohabitation de deux systèmes mythologiques distincts sera approchée à partir de la question de l'ancrage national et la dimension politique octroyées aux mythologies depuis le $\mathrm{XIX}^{\mathrm{e}}$ siècle tandis que la question du rôle de Kratos dans le panthéon nordique sera analysée grâce à certains des outils développés par Calame et Johnston dans leurs travaux sur la mythologie grecque. Ainsi nous essaierons de comprendre les choix narratifs des développeurs à partir de la confrontation des contextes intra - les récits qu'ils choisissent de présenter à travers les différents opus de la licence - et extra-discursif le contexte d'énonciation particulier que représente le jeu vidéo - tel que le propose Calame et les concepts de «monde-histoire» (story world) et de «réseau de personnages » (network of characters) utilisés par Johnston.

\section{Une cohabitation impossible : le(s) monde(s) et la(es) mythologie(s)}

La première réaction, liée à la question de la cohabitation de deux systèmes polythéistes dans un même espace, est particulièrement pertinente car elle révèle à la fois les spécificités des représentations des joueurs, de celles des choix narratifs de 
l'équipe de développement, ainsi que des principaux éléments du contexte intradiscursif de la série. Il s'agit d'abord du récit, inédit dans la mythologie grecque, sur lequel les premiers opus s'organisent : la montée en puissance, de l'humain au divin, de Kratos dont l'acmé réside dans le meurtre des principaux Olympiens. Ces morts s'accompagnent de modifications cosmologiques liées aux champs d'action des puissances divines disparues, déréglant le monde de Kratos et le rendant inhospitalier. Afin de prolonger la licence, les développeurs ont alors fait le choix de changer d'espace et d'emprunter l'esthétique d'un autre paysage mythologique.

Cohérent avec la dernière scène de God of War III ${ }^{22}$, ce choix narratif implique cependant une définition particulière de l'espace dans lequel évolue Kratos. Il faut que ses points de départ et d'arrivée soient reliés, via des liens physiques ou non, par leur inscription au sein d'un même espace - la Terre - afin de lui permettre de passer de l'un à l'autre. Toutefois, faire de ces deux points des lieux si distincts qu'ils soient, pour des sphères d'activité équivalentes, régis par des dieux différents, induit une Terre dont les régions ne sont pas unies par les mêmes principes de fonctionnement. C'est précisément à cette situation que réagissent certains joueurs :

Je ne sais pas s'il serait plus logique que les dieux grecs aient seulement dominé les Balkans / l'Europe du Sud et que les dieux celtiques, egyptiens, nordiques, mésopotamiens et autres leur soient contemporains, ou si à chaque époque, il y aient, des dieux universels mais situés à des endroits différents, qui apparaissent quand les précédents meurent. Les dieux seraient donc, dans cette deuxième hypothèse, omnipotents sur tout le globe mais se situeraient seulement dans la région qui leur " plaît». Toute l'histoire des Titans grecs est censée concerner tout le globe. Les Enfers grecs aussi. Donc pour moi la théorie «cyclique» est plus plausible. $^{23}$

Il est possible que les développeurs aient eu parfaitement conscience de ce qu'ils faisaient car l'organisation du monde à laquelle réagit ici le joueur nous apparaît suggérée tout au long de la narration. ${ }^{24}$ Selon nous, la quête des protagonistes, en accord avec les potentialités de la mythologie nordique, les font traverser plusieurs "mondes» possédant chacun des caractéristiques différentes représentées, entre autres, par des différences climatiques: Midgard, Alfheim, Muspelheim, Helheim, Jötunheim, Niflheim. Toujours à partir de ce paysage mythologique, ces différents mondes sont accessibles, car ils appartiennent tous au même espace, figuré par l'arbremonde Yggdrasil. En ayant recours à son pouvoir, il est possible de voyager entre des lieux possédant leurs propres règles. L'écart qui réside entre ces différents territoires est exprimé à travers le vocabulaire choisi par les développeurs; en effet, les différents "mondes » où évoluent les héros sont tous désignés en anglais sous le terme de realm [royaume], et non de world [monde]. Realm semble avoir un sens légèrement différent de world car il est dérivé, comme le français " règne ", du nom latin regimen, substantif du verbe rego (Ernaut \& Meillet, 1932, p. 567-569), signifiant l'action de diriger en droite ligne. Plus largement, dans son acception moderne, le terme « royaume » renvoie à une partie de la totalité que tend à désigner le mot « monde ».

Choisir d'user du premier mot implique que les espaces nommés comme tels détiennent leurs propres principes directeurs, suggestion qu'on retrouve également dans le mot français « règne", en particulier lorsqu'il est appliqué à des types d'êtres vivants : le règne animal ou végétal. En version originale, Kratos et son fils Atreus passent alors de royaume en royaume grâce à des voyages entre les branches de l'arbre-monde. Ainsi, l'espace (world) où ils évoluent est décrit comme abritant des territoires (realms) qui, 
bien que très distants, littéralement comme figurativement, au point de reposer sur des principes de fonctionnement différents, sont reliés, autorisant de fait le passage de l'un à l'autre. Une construction qui nous paraît difficilement superposable à la cosmographie mythique nordique où, si le suffixe -heimr (Cleasby, 1962 [1857], p. 251) renvoie bel et bien à des espaces précis censés être le foyer d'entités spécifiques à l'image de Jötunheim où résident les géants (p. 251), et donc proche des termes realm et « royaume », ce n'est pas le cas du suffixe -gardr. Étymologiquement, ce dernier renvoie à l'idée d'« espace clos » (p. 192) et ne possède pas la dimension englobante des termes anglais world ou français « monde ». Un gardr ne semble pas pouvoir contenir des heimr comme un monde peut contenir des royaumes comme semble l'indiquer l'existence de plusieurs espaces portant ce nom, Asgardr, le lieu clos des Ases, Midgardr, le lieu clos intermédiaire et Utgardr, le lieu clos de l'extérieur (p. 192).

Par conséquent, l'espace figuré par l'arbre-monde semble être l'équivalent de la Terre et la Grèce, un autre des royaumes visitables - une autre partie de l'arbre-monde puisque sa destruction initiée par Kratos n'atteint aucunement Midgard. Mais c'est sans compter sur la créativité des développeurs et leur volonté de respecter un certain contexte intra-discursif, à savoir le récit de la paternité de Kratos. Car l'un des thèmes principaux de cet opus étant le rapport de Kratos à son passé en lien avec l'identité de son fils ${ }^{25}$, les concepteurs ont placé au cœur de la narration le souvenir de son lieu de départ. De fait, lorsque le joueur pénètre dans la tombe du dieu Tyr, les protagonistes découvrent un trésor constitué de richesses provenant des quatre coins de la Terre. Parmi les territoires représentés figure la Grèce, incarnée, entre autres, par un emblème stéréotypé de sa société : l'amphore de vin. Kratos décide de la partager avec son fils afin de lui en apprendre un peu plus sur son passé. On apprend ainsi que le vin et Kratos viennent du même endroit, qui est nommé land en anglais, et traduit par " terre » au sens de région. Dans la mesure où aucun élément dans le jeu ne permet de supposer que la Grèce fait partie de l'arbre-monde ou est une région de Midgard, il semble qu'il existe des territoires en dehors d'Yggdrasil, mais qui appartiennent au même espace que lui et les royaumes qu'il connecte.

L'élément du jeu qui illustre le mieux cette construction est le personnage de Tyr, présenté, entre autres, comme un voyageur, tels que l'indiquent les effets retrouvés dans son tombeau. Ils permettent d'en faire un dieu ayant visité de nombreuses terres, lands, éloignées de son territoire d'origine, indiqué comme étant Midgard, où il partageait son savoir avec les humains. Les artefacts présents dans sa tombe laissent penser qu'il a voyagé dans des territoires où se sont développés des polythéismes célèbres : Grèce, Égypte et Japon. L'importance de Tyr repose donc sur le fait qu'il est celui qui unifie les deux paysages mythologiques et rend plausible le voyage de Kratos. À travers lui, c'est toute une organisation de l'espace qui se dévoile. Il suggère l'existence d'un espace uni, mais comprenant de multiples subdivisions qui, bien que reliées, sont assez distantes pour présenter des écarts dans leurs principes de fonctionnement.

Derrière cette construction cosmographique choisie par les développeurs, et à laquelle certains joueurs adhèrent, apparait une certaine conception de la mythologie. Une conception nationale ou "romantique» (Di Filippo, 2016b, p.126, 2020; Panaro, 2019 p. 15-18) en référence à son apparition au xIX siècle où l'étude de la mythologie était largement influencée par des objectifs idéologiques nationalistes. Chaque mythologie était alors attachée à l'histoire d'un peuple en particulier et servait, en 
témoignant de son ancienneté, à construire son identité nationale et sa supériorité par rapport aux autres comme l'a montré Bruce Lincoln (1999) ${ }^{26}$ à partir de l'analyse du discours scientifique sur la mythologie indo-européenne et aryenne. Il s'agit d'une conception qui a encore cours aujourd'hui et qui semble avoir débordé le cadre scientifique et nourrir les représentations populaires d'une mythologie (Von Schnurbein, 2016 ; Meylan, Rösli, 2020).

C'est à cette organisation du monde et la définition d'une mythologie qu'elle induit, que s'opposent les joueurs qui sont de l'avis de l'impossibilité pour plusieurs polythéismes de coexister sur un même espace. Selon eux, il est impossible que, sur la même terre, plusieurs puissances divines gouvernant la même sphère d'activité soient simultanément actives et, donc, régissent les mêmes phénomènes naturels. Au-delà des questions de définition de la mythologie, cette position nous parait reposer sur une organisation de l'espace spécifiquement propre à notre compréhension moderne fondée sur la science expérimentale voulant que nous évoluions dans un espace uni justement par la constance des lois réglant les phénomènes naturels, de sorte qu'ils aient chacun, partout et en tout point, les mêmes principes directeurs. ${ }^{27}$

\section{Kratos et le panthéon nordique ou la question de la cohérence de la matière mythique}

30 Le second thème principal qui ressort de l'analyse des fils de discussion est la question de l'identité de Kratos une fois intégré au panthéon nordique. Ici, c'est à travers les différents arguments utilisés par les internautes que se révèlent à la fois la tension au cœur de cette question et le nœud scénaristique sur lequel elle s'ancre. Selon nous, comprendre ce nœud nécessite de mesurer l'impact du contexte intra-discursif soustendant la série sur le paysage mythologique dont il est le support, comme, par exemple, celui du dernier volet.

31 Au fil des discussions, chaque internaute présente sa propre théorie, arguments à l'appui. Mais au-delà de leur contenu, leur intérêt réside dans les réponses qu'elles génèrent. En effet, le principal point de référence pour mettre en lumière l'identité de Kratos est le nom nordique d'Atreus (Loki) et/ou sa nature de Géant. Aussi, Atreus reçoit d'emblée une place dans le panthéon nordique sous l'identité de la divinité Loki, ce qui est beaucoup plus difficile pour Kratos. Les propositions et les réfutations sont très diverses, mais elles sont reliées par un même mode d'argumentation qui, le plus souvent, fait appel aux logiques des deux paysages mythologiques concernés. La principale de ces logiques est l'intrigue du Ragnarök que les développeurs choisissent de placer au cœur du dernier opus afin de dépeindre une nouvelle fois Kratos comme un "tueur de dieux» en l'insérant dans le récit traditionnel de la mort des principales divinités nordiques. C'est à la fin du jeu que la volonté des développeurs de raconter leur version de l'évènement devient explicite : après le meurtre de Baldr par Kratos survient un climat hivernal correspondant au Fimbulvetr, un hiver censé durer trois ans et être la première étape menant à la fin du règne des dieux. ${ }^{28} \mathrm{~A}$ partir de ces deux indices, les théories s'échafaudent, dont une particulièrement éloquente :

Je ne crois pas que la théorie Tyr =Kratos fonctionne. Cela va trop compliquer les choses avec beaucoup de paradoxes issus de voyages dans le temps et Kratos ne m'est jamais apparu comme un pacifiste attaché au respect de la culture. Je n'ai pas non plus d'idée sur la manière dont ils vont introduire Skoll/Hati dans la mesure où 
ils sont les petits-enfants de Loki. Si on parle des enfants de Loki, il faut aussi introduire Fenrir qui tue Odin et Hel qui est supposée régner sur Helheim. ${ }^{29}$ contradictions entre le récit proposé par les développeurs et celui, traditionnel, qu'appelle le nouveau paysage mythologique où évolue Kratos..$^{30}$ Cet internaute explique que, selon lui, Kratos n'est pas Tyr car si les développeurs comptent faire le récit du Ragnarök, où effectivement Loki détient une place prépondérante, l'incohérence guette. De fait, le rôle que Loki prend dans cet événement dépend de ses aventures passées en tant que divinité Ase. Par exemple, Fenrir, fils de Loki et de la géante Angrboda, est le père de Sköll, le loup à qui est attribué l'une des étapes essentielles du Ragnarök, la disparition du soleil ${ }^{31}$; puis, c'est ensuite Fenrir qui se libère et qui tue Odin. Fenrir, Jörmungandr (le serpent-monde) et Hel (gardienne du royaume des morts), tous enfants de Loki, prennent part à la chute des dieux..$^{32}$ Or, Kratos et Atreus rencontrent le serpent-monde au cours de leur périple. Pour cet internaute, le danger réside dans le fait d'avoir initié le Ragnarök alors que Loki n'est encore qu'un enfant et que la race des Géants ne compte plus qu'un représentant, Atreus lui-même, car pour que le récit soit cohérent, il faudrait que Loki soit grand-père. En conséquence, la narration se doit d'aménager un moyen pour qu'Atreus, même s'il l'a rencontré enfant, soit le père du serpent-monde.

L'internaute met ainsi en lumière le problème narratif majeur du fait d'avoir voulu, en sus du transfert d'un paysage mythologique à l'autre, donner à Kratos un rôle dans son paysage d'arrivée, celui du père d'une de ses principales divinités. Car en faisant d'Atreus Loki, les développeurs l'incluent dans un récit construit de façon cohérente avec sa propre temporalité et son intrigue que le récit de l'enfance de Loki perturbe. ${ }^{33}$ S'ils souhaitent réaligner leur narration sur le récit traditionnel du Ragnarök, ils devront alors recourir à des artifices scénaristiques qui ne plaisent pas à cet internaute.

Ce type de réaction nous apparaît tout à fait caractéristique de la manière dont certains joueurs pensent une mythologie. Comme l'a démontré Johnston, ce corpus de récits qu'on nomme aujourd'hui "mythologie " crée à travers le cumul de ses intrigues un « monde-histoire» (story world) ${ }^{34}$ (Johnston, 2018, p. 124-125 ; Panaro 2019 p. 18-20), un monde qui, au-delà des espaces construits par chacun des récits dans leur singularité, émerge et est rendu cohérent par le « réseau de personnages " (network of characters) qu'établit cette accumulation (Johnston, 2018, p.131). Les récits ont beau être multiples, comme leurs différentes versions, pris dans leur ensemble, ils figurent un monde où évoluent des personnages qui sont tous liés les uns aux autres, le plus souvent par des liens généalogiques. C'est avec cette toile de fond que les choix narratifs des développeurs entrent en conflit, induisant les critiques des joueurs les plus familiers des mondes-histoires et des réseaux de personnages que construisent les mythologies grecques et nordiques. Car la volonté des développeurs de raconter à la fois l'histoire d'un tueur de dieu, le contexte intra-discursif propre à l'ensemble de la licence, et celle, propre au dernier opus de celle-ci, d'un père élevant son fils, les a poussés à proposer une variation du mythe du Ragnarök tel qu'il est raconté, entre autres, dans la Völuspa. Ainsi, en prenant la décision de l'initier bien que Loki est encore un enfant, leur récit entre en contradiction avec le réseau de personnages qui soustend la mythologie nordique et fonde la cohérence du monde-histoire qu'elle construit. L'introduction de Kratos et son fils dans ce réseau fait alors émerger des incohérences 
que seuls les joueurs les plus familiers de la version traditionnelle du récit du Ragnarök identifient et relèvent, comme le résume très bien cet internaute :

C'est intéressant de se poser la question " comment l'auraient-ils insérée ? » [une autre mythologie] car au final l'histoire que voulait raconter Cory [Barlog] était cette histoire de la maturité père / fils et il n'y avait pas de bonne manière de la mettre en parallèle avec la mythologie nordique. Ils ont donc inventé des moyens de la mettre en parallèle - ils ont fait de Baldur un méchant qui voulait tuer sa mère et fait de Thor un père abusif qui bat son propre fils à mort. Nous avons donc des relations parent / enfants tordues qui contrebalancent la maturité de Kratos et Atreus, mais elles n'étaient pas du tout présentes dans le mythe - les histoires et les personnages ont dues être modifiés pour coller avec ce récit.

Le nœud des critiques réside donc dans l'apparente opposition de ce que savent les joueurs les plus renseignés sur la mythologie nordique et le récit mis en place par les développeurs. Pour ces joueurs, il existe un canon du récit du Ragnarök que le récit des développeurs ne respecte pas; une attitude qui nous apparait devoir être mise en lien avec le type de sources d'où ils tirent leurs connaissances. Ces sources ne sont pas les textes médiévaux comme l'Edda de Snorri, mais des reformulations modernes (à l'image de celle de Gaiman ou des sites de vulgarisation tel Wikipédia) où les auteurs ont fondu la matière mythique originale en un récit respectant les codes littéraires actuels (en particulier en termes d'unité de l'intrigue), renforçant au passage la cohérence de l'ensemble. De sorte que les critiques concernant le monde-histoire et le réseau de personnages révèlent ainsi certaines des attentes d'une partie des joueurs en termes de récit mythique. Pour accepter le transfert d'un paysage mythologique à un autre, ils souhaitent que la position d'arrivée de Kratos soit cohérente avec ce qu'ils connaissent des canons de la mythologie nordique (ici le récit du Ragnarök), qu'elle respecte ce qu'ils savent de cette dernière à partir d'œuvres littéraires modernes basées sur les Eddas. De fait, ils ne sont prêts à accepter des variantes d'un récit mythique que si celui-ci s'aligne sur leurs connaissances préalables, à l'image d'un « reliquat narratif » (Stierle, 1972, cité dans Paissa, 2016, \$30) au sens où il acquiert un «statut de figure figée fonctionnant comme un stéréotype mémoriel » qu'active toute référence à l'évènement (ici le Ragnarök).

Cette attente en termes de cohérence fonde, comme la question de son ancrage national, la spécificité de leurs représentations quant à ce que doit être une mythologie. Car les récentes recherches sur la mythologie nordique ont, au contraire, mis en évidence l'absence de cohérence dans les divers récits que nous livrent les sources médiévales (Sturluson, 1982, p. XXVI). A. Faulkes pense ainsi que la mythologie nordique est un «ensemble désorganisé de traditions conflictuelles» (cité et traduit par Di Filippo, 2016b, p. 122). Ce manque de cohérence tient principalement à la nature même de ces sources médiévales qui sont elles-mêmes de nature très diverses (Abram, 2011, p. 2). En effet, la quasi-totalité des textes retraçant les divers récits mythologiques nordiques ne datent pas de la période où la religion polythéiste à laquelle ils participent était encore pratiquée (Meylan, 2020). Il s'agit au contraire de textes rédigés dans un monde d'ores et déjà christianisé et par des auteurs qui le sont tout autant. Si donc l'Edda de Snorri Sturluson tend à présenter un corpus cohérent de récits mythiques, il ne s'agit pas d'un ouvrage à caractère religieux mais d'un manuel de poésie (Di Filippo, 2016b, p. 117). Aussi, la cohérence qui appert dans cet ouvrage est davantage à penser comme le résultat du travail de systématisation opéré par l'auteur plutôt qu'une caractéristique intrinsèque de la mythologie nordique (Di Filippo, 2016b, p.116). Ce que confirme l'étude d'autres sources, à l'image de la toponymie (Brink, 
2007, p. 124-125) qui montre que la grande variété des dieux présentés par les récits mythologiques ne se retrouvent pas dans les lieux de culte qui s'organisent autour d'un nombre très restreint de dieux (Odin, Thor et Freyr au premier chef). Plus largement, le manque de cohérence au sein du corpus mythologique nordique est à comprendre à partir de la multiplicité des contextes locaux d'apparition de ces mythes (Gunnell, 2015, p.57). Chaque communauté semble, par conséquent, avoir développé ses propres versions de récits mythiques selon leurs caractéristiques propres, jusqu'à privilégier d'autres hiérarchies divines que celle présente chez Snorri avec Odin à son sommet (Gunnell, 2018, p. 105-129).

Si la question de la cohabitation de plusieurs systèmes mythologiques dans un même espace a révélé la pénétration des théories romantiques et nationalistes dans les représentations populaires d'une mythologie, celle de la place de Kratos dans le panthéon nordique souligne celle des théories structuralistes. Les joueurs les plus renseignés abordent alors, à l'image de chercheurs structuralistes $\mathrm{du} \mathrm{xx}^{\mathrm{e}}$ siècle tels que Georges Dumézil, la mythologie comme une matière cohérente. À terme, la question de la représentation sociale de la mythologie chez les joueurs de God of War fait apparaître la mécanique d'élaboration de connaissances "populaires » à partir de discours scientifiques tels que l'ont déjà mis en exergue Miles Hewstone et Serge Moscovici $(1983 ; 1984)$.

\section{Conclusion}

Le forum est un outil précieux pour une première approche de l'étude des représentations des joueurs sur la mythologie nordique. Du fait de son statut de métajeu, il offre un espace où la trame et les articulations du jeu peuvent être transposées, discutées et critiquées de manière collective. On a pu voir que peu importait la langue des joueurs, les stratégies argumentatives pour se saisir du sujet et intervenir étaient communes : avis personnel dénué de support externe ou au contraire renvoyant à un autre jeu ou bien à de la littérature académique ou non. Dans ce dernier cas, la mise en série des messages permet de se rendre compte que les textes médiévaux scandinaves ne servent principalement de références normatives qu'à travers le monde qu'elles construisent et non pour leur texte stricto-sensu. Bien plus nombreux sont ceux qui se tournent plutôt vers des versions contemporaines et romancées des Eddas en particulier.

39 L'étude des différents fils de discussion a permis de mettre en lumière deux pôles principaux de réactions au changement de paysage mythologique entre les deux derniers opus de God of War. Le premier renvoie à l'impossibilité pour certains joueurs de voir coexister deux polythéismes dans un même espace. Ceux qui refusent la plausibilité de cette cohabitation s'adossent à la vision d'un monde dont les différentes parties trouvent leur unité dans les principes directeurs qu'elles partagent toutes, appliquant alors à celui-ci une vision scientifique et/ou monothéiste induisant une définition totalisante de la mythologie. Au contraire, ceux qui voient une possibilité à cette coexistence fondent leur avis sur l'idée qu'une mythologie est définie par son inscription dans un territoire précis et s'y confine. On retrouve alors en filigrane chez ces derniers la même définition romantique et nationaliste de la mythologie qui était soutenue au $\mathrm{xIX}^{\mathrm{e}}$ siècle, voulant que chacune s'attache à l'histoire d'un peuple en 
particulier et dans un espace restreint. Ce sont donc deux visions du monde qui s'opposent dans la définition même du terme.

Le second pôle de réactions s'articule autour de l'introduction par les développeurs de Kratos et Atreus au sein du panthéon nordique et de leur rôle dans le déroulement du Ragnarök. En effet, c'est au prix de plusieurs entorses au texte de la Völuspa que ces derniers les intègrent dans une histoire déjà écrite. Atreus ayant été identifié à Loki par les concepteurs, les joueurs ne manquent pas souligner l'incohérence qui émerge au sein du réseau de personnages et plus largement du monde-histoire construit par les récits traditionnels. Leurs réactions traduisent leurs attentes en termes de cohérence en matière de récit mythologique. Pour qu'ils adhèrent à la narration proposée par les développeurs, ces joueurs s'attendent à ce qu'elle se superpose parfaitement avec leurs propres connaissances de la mythologie nordique et des logiques internes assurant sa cohérence. Toutefois, leur savoir apparaît lui-même en décalage avec les travaux académiques actuels qui ne cessent de démontrer que cette dernière est dénuée de cohérence et est plutôt à comprendre à partir de son ancrage local. L'impératif de cohérence que ces joueurs attachent aux récits mythiques rappelle en revanche fortement l'approche structuraliste des anthropologues $\mathrm{du} \mathrm{xx}^{\mathrm{e}}$ siècle pour qui la dimension narrative des mythologies humaines était au premier plan et sur laquelle était basée leur supposée cohérence.

41 Les réactions des joueurs à ce changement de paysage mythologique et plus généralement leurs représentations de la mythologie nordique semblent ainsi trouver leurs racines dans un discours scientifique non actualisé. En ce sens, nos conclusions rejoignent celles qu'énonçaient Di Filippo (2019) dans son article concernant l'usage des représentations vikings médiévales dans le jeu Advanced Dungeons \& Dragons. Leur étude permet de faire émerger une question importante qui est celle de la manière dont les connaissances scientifiques infusent dans la culture populaire. On voit très bien ici qu'il n'y a pas d'évolution rapide des représentations mythologiques en fonction des dernières recherches académiques mais plutôt une évolution différée qui passe par des intermédiaires que sont la littérature contemporaine ou le jeu vidéo qui usent euxmêmes de représentations anciennes. Les travaux des chercheurs semblent encore trop méconnus pour pouvoir participer directement à la construction d'une représentation actualisée de la mythologie nordique. Toutefois, cette conclusion n'est applicable qu'aux joueurs dans la mesure où il existe des jeux pour lesquels des chercheurs ont été intégrés à l'équipe de développement tels qu'Assassin's creed Valhalla (2020). Par conséquent, il est possible qu'il existe des cas où les représentations correspondent au discours scientifique le plus actuel.

Mais en ce qui concerne God of War, des romanciers tels que Gaiman ou Harris, pour qui les mythologies sont avant tout des supports narratifs, ou encore des développeurs comme Barlog qui au-delà du narratif, leur empruntent également des éléments scénaristiques et esthétiques, apparaissent par conséquent comme des passeurs entre ces deux mondes, des agents du processus de «travail sur le mythe » mis en évidence par Blumenberg (2001 [1979]). C'est cette présence d'intermédiaires qui, selon nous, induit une transmission différée du savoir académique et donc des représentations populaires déphasées (Di Filippo, 2019). Si ici nous avons usé de l'étude d'un cas particulier, il faudra à l'avenir élargir l'enquête à d'autres communautés de joueurs mais aussi d'autres espaces comme l'Asie par exemple. On pourra alors chercher à confronter les deux terrains afin de déterminer les similitudes et les différences de 
leurs représentations de la mythologie nordique dans l'objectif d'augmenter le nombre de variables à considérer (Becker, 2016, p. 26).

\section{BIBLIOGRAPHIE}

ABRAM C. (2011) Myths of the Pagan North. The Gods of the Norsemen, New York, Continuum.

AMOSSY R. (2012) «Faut-il intégrer l'argumentation dans l'analyse du discours ? Problématiques et enjeux ", Argumentation et Analyse du Discours, 9, https://journals.openedition.org/aad/1346.

BAINBRIDGE (2013) eGods: Faith Versus Fantasy in Computer Gaming, New York, Oxford University Press.

BATESON G. (1977) Vers une écologie de l'esprit, Paris, Seuil.

BECKER H. (2016) La bonne focale. De l'utilité des cas particuliers en sciences sociales, Paris, La Découverte.

BIANCHI U. (1966) « Péché originel et péché “antécédent" ", Revue de l'histoire des religions, n², pp. 117-126.

BLUMENBERG H. (2001 [1979]) Arbeit am Mythos, Frankfurt am Main, Suhrkamp.

BOAS F. (1914) « Mythology and Folk-Tales of the North American Indians », The Journal of American Folklore, 106, pp. 374-410.

BOSMAN F. (2016) «The Word Has Become Game: Researching Religion in Digital Games », Online - Heidelberg Journal of Religions on the Internet, 11, pp. 28-45, https://heiup.uni-heidelberg.de/ journals/index.php/religions/article/view/23626

BORGEAUD P. (2010) « Mythes » in R. Azria, D. Hervieu-Léger et D. Iogna Prat (dirs.) Dictionnaire des faits religieux, Paris, Presses universitaires de France, pp. 855-861.

BOYER R. (1992) L'Edda poétique, Paris, Fayard.

BRINK S. (2007) « How Uniform was the Old North Religion? », in J. Quinn, K. Heslop et Wills T. (eds.) Learning and Understanding in the Old Norse World: Essays in Honour of Margaret Clunies Ross, Turnhout, Brepols, pp. 105-136.

CALAME C. (2015) Qu'est-ce que la mythologie grecque ?, Paris, Gallimard, 2015.

CAMPBELL H. et PRICE GRIEVE G. (2014) Playing with Religion in Digital Games, Bloomington \& Indianapolis, Indiana University Press.

CHAKER R. (2017) « Les espaces de discussion sur Internet comme dépassement des communautarismes? Le cas de l'agora virtuelle libanaise ", Éducation et socialisation, $n^{\circ} 43$, https://journals.openedition.org/edso/1996.

CLEASBY R. (1962 [1857]) An Icelandic-English Dictionary, Oxford, The Clarendon Press.

COOPER V. (2016) Fantasies of the North: Medievalism and Identity in Skyrim, Thèse de doctorat, Université de Leeds.

DE SARDAN J.-P. O. (1988) «Émique », L'Homme, n¹47, pp. 151-156. 
DETIENNE M. (1981) L'Invention de la mythologie, Paris, Gallimard.

DI FILIPPO L. (2016a) Du mythe au jeu. Approche anthropo-communicationelle du Nord. Des récits médiévaux scandinaves au MMORPG Age of Conan : Hyberborian Adventures, thèse de doctorat, Université de Lorraine.

DI FILIPPO L. (2016b) « Des récits médiévaux scandinaves aux mythes nordiques : catégorisation et processus d'universalisation », in C. Delaporte, L. Graser et J. Péquignot (eds.) Penser les catégories de pensée. Arts, cultures et médiations, Paris, L'Harmattan, p. 115-133.

DI FILIPPO, L. (2019) « La mise en scène ludique de l'Histoire : l'époque viking comme cadre de jeu pour Advanced Dungeons and Dragons. ", in A. Besson (dir.) Fantasy et Histoire, Épinal, Chambéry, Publications des Imaginales, pp. 391-414.

DI FILIPPO L. (2020) «Stereotype of the North in a Massively Multi-Player Role-Playing Game », in Meylan N. et Röstli L. (eds.) Old Norse Myths as Political Ideologies, Turnhout, Brepols.

DUSSARPS C. (2020) « Le jeu vidéo médiateur de savoirs en histoire : l'exemple de Crusader King 2 et Europa Universalis 4 », Sciences du jeu, 13, https://journals.openedition.org/sdj/2696.

ERNOUT A., MEILLET A. (1932) Dictionnaire étymologique de la langue latine : histoire des mots, Paris, Klinslieck.

FLICHY P. (2008) «Internet et le débat démocratique », Réseaux, n¹50, pp. 159-185.

GAIMAN N. (2017) La mythologie nordique, Vauvert, Au Diable Vauvert.

GUNNELL T. (2015) « Pantheon, what Pantheon? Concept of a Family of Gods in Pre-Christian Scandinavian Religions », Scripta Islandica, n66, 2015, pp. 55-76.

GUNNELL T. (2018) « How High was the High One? The Roles of Oðinn and Pórr in Pre-Christian Icelandic Society ", in S. Brink et L. Collinson, Theorizing Old Norse Myths, Turnhout, Brepols, pp. 105-129.

HARRIS J. (2014) The Gospel of Loki, Londres, Gollancz.

HEWSTONE M., MOSCOVICI S. (1983) « Social representations and social explanations: from the naïve to the amateur scientist », in M. Hewstone (ed.) Attribution Theory, Oxford, Blackwell, pp. 98-126.

HEWSTONE M., MOSCOVICI S. (1984) « De la science au sens commun », in S. Moscovici (ed.) Psychologie sociale, Paris, Presses universitaires de France, pp. 539-566.

ISER W. (1985 [1976]) L’Acte de lecture. Théorie de l'effet esthétique, Bruxelles, Mardaga.

JAUSS H. (2019 [1978]) Pour une esthétique de la réception, Paris, Gallimard.

JENKINS H. (2009) « The Revenge of the Origami Unicorn: Seven Principles of Transmedia Storytelling (Well, Two Actually. Five More on Friday) ", Confessions of an Aca-Fan: The Official Weblog of Henry Jenkins, consulté le 12 décembre 2018, http://henryjenkins.org/blog/2009/12/ the_revenge_of_the_origami_uni.html.

JOHNSTON S. I. (2018) The Story of Myth, Cambridge-London, Harvard University.

KOREN R. (2008) « Pour une éthique du discours : prise de position et rationalité axiologique », Argumentation et Analyse du Discours, 1, https://journals.openedition.org/aad/263.

LINCOLN B. (1999) Theorizing Myths. Narrative, Ideology, and Scholarship, Chicago, Chicago University Press. 
MEYLAN N. (2020) Religion, mythes et politique en Islande médiévale, Liège, Presses universitaires de Liège.

MEYLAN N. et RÖSLI L. (2020) Old Norse Myths as Political Ideologies, Turnhout, Brepols.

MOSCOVICI S. (1961) La psychanalyse. Son image et son public, Paris, Presses universitaires de France.

MOZELIUS, P., FAGERSTRÖM, A. et SÖDERQUIST, M. (2017) « Motivating factors and tangential learning for knowledge acquisition in educational games », The electronical journal of e-learning, 4 , pp. 343-354.

PAISSA P. (2016) «Exemple historique et argumentation : autour de la reconnaissance du massacre du 17 octobre 1961 », Argumentation et Analyse du Discours, 16, https:// journals.openedition.org/aad/2160.

PANARO Luca A. (2019) The "New" North. Perceptions, Projections and Memories of Medieval Scandinavia in Video Games as Popular Media, mémoire de recherche, Université d'Oslo / Université d'Islande.

PLEDEL I. (2007) « Le blog face à la clôture informationnelle : la délibération au service de l'apprentissage ", Distances et Savoirs, vol. 5, pp. 275-288, https://ds.revuesonline.com/article.jsp? articleId $=10696$.

PEETERS H. et CHARLIER P. (1999) «Contributions à une théorie du dispositif », Hermès, n²5, pp. $15-23$.

SAEMMER A. (2016) « Esthétique de la réception », Publictionnaire. Dictionnaire encyclopédique et critique des publics, http://publictionnaire.huma-num.fr/notice/esthetique-de-la-reception/

SCHAEFFER J.-M. (1999) Pourquoi la fiction?, Paris, Seuil.

STURLUSON S. (1982) Edda. Prologue and Gylfaginning, Oxford, Clarendon Press.

STURLUSON S. (1991) L'Edda. Récits de mythologie nordique, Paris, Gallimard.

TOLKIEN J. R. R. (1947) « On Fairy-Stories », in C. S Lewis (ed.) Essays Presented to Charles Williams, Oxford, Oxford University Press, pp. 38-89.

VERNANT J. P. (1962) Les origines de la pensée grecque, Paris, Presses universitaires de France.

VERNANT J. P. (1974) Mythe et société en Grèce ancienne, Paris, Maspero.

VEYNE P. (1983) Les Grecs ont-ils cru à leurs mythes? Essai sur l'imagination constituante, Paris, Seuil.

VON SCHNURBEIN S. (2016) Norse Revival: Transformations of Germanic Neopaganism. Leiden, Boston, Brill.

WAGNER R. (2012) Godwired. Religion, Ritual and Virtual Reality, Londres, Routledge.

WOLF W. (2013) « Aesthetic Illusion », in W. Wolf, W. Bernhart et A. Mahler (éds) Immersion and Distance: Aesthetic Illusion in Literature and Other Media, Amsterdam, Rodopi, pp. 1-63.

ZABBAN V. (2009) « Hors jeu ? Itinéraires et espaces de la pratique des jeux vidéo en ligne (enquête) ", Terrains \& travaux, n 15, pp. 81-104, https://www.cairn.info/revue-terrains-ettravaux-2009-1-page-81.htm?ref=doi.

ZABBAN V. (2014) «Les enjeux du métajeu. Les pratiques d'un jeu en ligne et leurs médiations. », in M. Atallah, P. Nova, M. Pellet et C. Indermuhle (éds) Pouvoirs des jeux vidéo. Des pratiques aux discours, Paris, inFolio, pp. 13-32. 


\section{NOTES}

1. Les auteurs tiennent à vivement remercier Selim Krichane, Yannick Rochat et Fabián Ruz pour leurs conseils à l'origine de cet article.

2. Voir par exemple les articles du site web Gamerblog des 3 mai 2018 et 21 mai 2019, https:// bit.ly/33tej7t et https://bit.ly/3f4gslv.

3. Telles que Denise Jodelet (2003 [1989], p. 53-54) les définit.

4. « Djigit », 17/02/2018, fil : "Chaque région du monde a ses propres dieux? », créé en Janvier 2018, Jeuxvideo.com, dernière consultation le 13 mars 2018, https://bit.ly/2xB2kbh.

5. Nous traduisons, «CapopaulieWalnuts », 02/01/2019, fil : «What Religions Don't Exist in God of War? », créé en janvier 2019, Reddit, dernière consultation le 13 mars 2020 [archivé], https:// bit.ly/2QrOGOT.

6. Tous les messages de joueurs présentés sont reproduits en conservant leur orthographe et leurs formes grammaticales originales.

7. «NaelVanDarnus ", 24/01/2018, fil : "Chaque région du monde... ». Cette idée est également discutée dans ce fil: "Coexistence Mythologie grecque et nordique impossible?", créé en Octobre 2018, Jeuxvideo.com, dernière consultation 30 octobre 2020, https://bit.ly/2HSsyvc.

8. « Xenoform », 06/02/2018, fil : « Chaque région du monde... ».

9. «Vafthrudnismal », Strophe 37 (Boyer 1992, p. 524-525).

10. « Völuspá », Strophes 42-43 (Boyer, 1992, p. 543).

11. "ImBurningStar_IV », 09/08/2018, fil: "Is the Giant bird in God of War the same shown in Hellblade Sensua's Sacrifise? ", créé en Août 2018, Reddit, dernière consultation 30 octobre 2020 [archivé], https://bit.ly/3oJ5tfi.

12. "Malcom89 ", " God of War fait le plein d'informations inédites ", Jeuxvideo.com, 05/03/2018, https://bit.ly/2S7EaNq.

13. "La Bhagavad Gìtā, «Chant du Bienheureux Seigneur", est un des longs passages spéculatifs insérés dans le Mahābhārata, la grande Épopée indienne, mais sa composition et son inspiration en font un tout autonome. Elle expose l'enseignement métaphysique et mystique donné par Viṣnu, sous la forme de Kṛșnạ, à l'un des princes, héros de la guerre qui va mettre aux prises deux clans rivaux et apparentés. » Anne-Marie ESNOUL, « BHAGAVAD GĪTĀ », Encyclopcedia Universalis, consulté le 01/02/2020, http://www.universalis.fr/encyclopedie/bhagavad-gita/.

14. « Sirliftalot35 », 02/01/2019, fil: « What Religions Don't Exist... ».

15. Voir par exemple les fils suivants : «Les gars... Imaginez un GOW basé sur la Mythologie CHRÉTIENNE?!», créé en Avril 2018, Jeuxvideo.com, dernière consultation 30 octobre 2020, https://bit.ly/2TKLKNL ; « What Religions Don't Exist... ».

16. On le retrouve par exemple ici: «Just as god of war 1-3 got me interested in Greek mythology, this game has managed to get me interested in Norse mythology ", créé en Avril 2018, Reddit, dernière consultation 30 octobre 2020 [archivé], https://bit.ly/3mFbjN0.

17. Voir notamment le fil: " Just wanted to show my book that I got today, playing God of War really made me thirsty for more Norse Mythology (+ I really like Neil Gaiman) », créé en Mars 2020, Reddit, dernière consultation 30 octobre 2020 [archive] , https://bit.ly/2JbGWiv.

18. Dans le fil suivant: «Just got this I'm gonna brush up on my norse mythologys before god of war 5 ", créé en Juin 2020, Reddit, dernière consultation le 30 octobre 2020, https://bit.ly/ 34FuEao.

19. « damei », 02/05/2018, fil : "Vous avez des livres à lire sur les mythologies? », créé en Mai 2018, Jeuxvideo.com, dernière consultation 30 octobre 2020, https://bit.ly/2TKLtdH..

20. Poème scaldique anonyme ( $\mathrm{x}^{\mathrm{e}}-\mathrm{XI}^{\mathrm{e}}$ siècles) qui évoque l'histoire du monde depuis ses origines jusqu'à sa destruction dont Snorri Sturluson reprend une partie dans son Edda.

21. Très proche de ce que Jean-Marie Schaeffer (1999, p. 327-335) a nommé « feintise ludique ». 
22. La fin de God of War III montre, à l'endroit où devrait reposer le cadavre de Kratos après son affrontement avec Zeus, une traînée de sang évoquant la fuite du héros.

23. «Profil supprimé ", 24/01/2018, fil : « Chaque région du monde... ».

24. C'est ce que semble confirmer Cory Barlog lorsqu'il indique que chaque "mythologie» possède son propre territoire. Voir l'interview donnée au site Kotaku le 05/01/2018, https:// bit.ly/2GcnUYb.

25. Un dieu qui s'ignore et qui est élevé comme un humain.

26. On peut également penser au texte d'Eugène Sue, Les mystères du peuple (1849), qui insiste sur les origines gauloises fantasmées des "prolétaires" français face à une bourgeoisie d'origine romaine aux lendemains de la révolution de 1848.

27. Comme en contexte polythéiste où à chaque puissance divine correspond un champ d'action donné alors qu'en contexte monothéiste une seule et même puissance divine régit l'entièreté de ces phénomènes naturels sur la surface de la Terre.

28. Chapitre 51 de la « Gylfaginning » de l'Edda de Snorri (Sturluson, 1991, p. 95).

29. "Birador ", 05/01/2020, fil: " Did Baldur know Kratos was a God from the Beginning? (Or even at all) », créé en Janvier 2020, Reddit, dernière consultation le 13 mars 2020, https://bit.ly/ 2wPAwQ2.

30. Caractéristique soulevée également dans ce fil : «Kratos is *spoiler*?», créé en Mai 2018, Reddit, dernière consultation le 13 mars 2020 [archivé], https://bit.ly/2U35iwH.

31. «Völuspa », strophe 40, (Boyer, 1992, p. 543).

32. La paternité de Loki est évoquée dans le chapitre 34 de la «Gylfaginning " de l'Edda de Snorri et la bataille du Ragnarök est racontée dans le chapitre 51, (Sturluson, 1991, p. 61 ; p. 95-99).

33. Cela est aussi discuté dans le fil suivant: « How correct is God of Wars Norse mythology? ", créé en Janvier 2019, Reddit, dernière consultation 30 octobre 2020, https://bit.ly/31Wl5SX.

34. À la manière dont Tolkien (1947, p. 38-89) parlait des « secondary worlds » développés par les œuvres de fantasy.

\section{RÉSUMÉS}

En 2018, le studio Santa Monica sort un nouvel opus de sa licence God of War dans lequel, contrairement aux jeux précédents, son héros Kratos n'évolue plus en Grèce mais en Scandinavie et est cette fois aux prises avec un nouveau panthéon. Dans cet article, nous nous intéressons aux représentations de la mythologie chez les joueurs à partir de leurs réactions à ce passage d'un paysage mythologique à l'autre. Nous nous appuyons pour ce faire sur l'analyse de plusieurs discussions menées sur deux forums entre 2018 et 2020, Jeuxvideo.com et Reddit. La lecture des différentes conversations laisse en effet entrevoir d'importants débats sur la plausibilité narrative du passage d'une mythologie à l'autre et sur les incohérences engendrées par l'arrivée de Kratos au sein d'un nouveau panthéon. À la suite de notre étude, il apparaît clairement que les joueurs fondent leurs représentations de la mythologie non pas sur les travaux académiques les plus récents, mais à partir de media intermédiaires eux-mêmes vecteurs de représentations altérées.

In 2018, the Santa Monica studio released a new God of War. Contrary to the previous games, its hero Kratos no longer plays in Greece but in Scandinavia with a new pantheon. In this article, we are interested in player's mythology representations from their reactions to this passage from 
one mythological landscape to another. We draw on several discussions held on two forums between 2018 and 2020, Jeuxvideo.com and Reddit. Reading the different conversations reveals important debates about the narrative plausibility of the transition from one mythology to another and the inconsistencies generated by the arrival of Kratos in a new pantheon. Following our study, it is clear that the players base their representations of mythology not on the most recent academic works, but on intermediate media which are themselves vectors of altered representations.

\section{INDEX}

Keywords : God of War, Scandinavia, forum, mythology, metagame, representations

Mots-clés : God of War, Scandinavie, forum, mythologie, métajeu, représentations

\section{AUTEURS}

\section{ADRIEN COIGNOUX}

Université de Paris - Anthropologie et Histoire des Mondes Antiques (ANHIMA)

\section{YOHANN GUFFROY}

Laboratoire d'Histoire des Sciences et des Techniques (LHST), École polytechnique fédérale de Lausanne (EPFL) 